\title{
Transition-metal-oxide based functional thin-film device using leakage-free electrolyte
}

\author{
Takayoshi KATASE ${ }^{*, * *, * *, \dagger}$ and Hiromichi OHTA ${ }^{* *, \ddagger}$ \\ *Laboratory for Materials and Structures, Tokyo Institute of Technology, 4259 Nagatsuta, Midori, Yokohama 226-8503, Japan \\ ${ }^{* *}$ Research Institute for Electronic Science, Hokkaido University, N20W10, Kita, Sapporo 001-0020, Japan \\ *** PRESTO, Japan Science and Technology Agency, 7 Gobancho, Chiyoda, Tokyo 102-0076, Japan
}

\begin{abstract}
Using the flexible valence states of transition-metal-oxides (TMOs), the optical, electronic, and magnetic properties can be simultaneously controlled by electrochemical oxidation and reduction. Herein we review our recent works on the electrochemically switchable functional thin-film device, which has a three-terminal thin-film-transistor (TFT) structure with the TMO as an active channel layer and the liquid-leakage-free electrolyte as a gate insulator. Thin films of vanadium dioxide, tungsten trioxide, and strontium cobaltite were selected as the channel layer. By applying the gate voltage at room temperature in air, the protonation/ deprotonation or oxidation/deoxidation occurs in the TMO channels and their opto-electronic and electro-magnetic properties are reversibly switched by using the mobile ions in the solid TFT structure. The present device with liquid-leakage-free electrolyte provides a novel design concept for the development of future multifunctional switching devices based on TMOs.
\end{abstract}

(C2017 The Ceramic Society of Japan. All rights reserved.

Key-words : Transition metal oxide, Thin film transistor, Liquid-leakage-free electrolyte, Electrochemical reaction, Multifunctional switching device

[Received April 29, 2017; Accepted May 23, 2017]

\section{Introduction}

Transition metal oxides (TMOs) have received a great attention as an active material for future functional devices because of their many switchable physical properties, such as electrical conductivity, optical absorption, magnetism, and superconductivity. ${ }^{1)-3)}$ The rich variety of the functional properties in TMOs originates from the flexible valence states of TM ions. ${ }^{4)}$ Depending on the number of $d$-electrons in the TM ions, the electronic structure of TMOs dramatically changes and the consequent giant physical responses are attractive for the development of novel switching devices.

One of the effective ways to control their functions is the use of electrostatic field effect. By using the thin film transistor (TFT) structure, the carrier concentration at the TMO channel surface can be controlled by applying the external gate voltage. An electric field generally up to $\sim 10 \mathrm{MV} \mathrm{cm}^{-1}$ can be applied to active channel through gate dielectrics, ${ }^{5)}$ such as amorphous (a-) $\mathrm{SiO}_{2}$ (dielectric constant, $k=3.9)$, a- $\mathrm{Al}_{2} \mathrm{O}_{3}(k=9.0)$, a- $\mathrm{Y}_{2} \mathrm{O}_{3}(k=$ 15.0) and a- $\mathrm{HfO}_{2}(k=25.0){ }^{6}{ }^{6}$ However, the modulation range of sheet carrier concentration is limited to $\sim 10^{13} \mathrm{~cm}^{-2}$ due to their low breakdown field of such dielectric oxides and cannot exceed the critical value of $\sim 10^{14} \mathrm{~cm}^{-2}$, where the TMOs exhibit dramatic changes in the optical, electronic, and magnetic properties through phase transitions. ${ }^{7}$ )

In order to overcome this limitation, electric double layer transistor (EDLT) configuration has been employed, where the electrolyte solution is used as a gate dielectric. The large capacitance of polarized ions in a liquid electrolyte or ionic liquid can be used to accumulate high-density sheet carrier concentration

Corresponding author: T. Katase; E-mail: katase@mces.titech. ac.jp

* Corresponding author: H. Ohta; E-mail: hiromichi.ohta@es. hokudai.ac.jp up to $\sim 10^{15} \mathrm{~cm}^{-2}$ and the EDLT has been proved to be a powerful approach to modulate the electronic states in a very wide range. ${ }^{8)-11)}$ However, the electrostatic approach using EDLT can not be applied for the practical applications without sealing and the modulatable thickness is limited in the screening length.

Another approach to control the functions of TMOs is the modification of their non-stoichiometry, i.e. the control of oxygen excess or deficiency and protonation, because the valence states of TM ions in TMOs are quite variably dependent on the oxygenanion off-stoichiometry and their coordination conditions. ${ }^{12), 13)}$ For example, tungsten trioxide $\left(\mathrm{WO}_{3}\right)$, known as an electrochromic material, is a transparent insulator, but it becomes a blue colored metal by the protonation $\left(\mathrm{H}_{x} \mathrm{WO}_{3}\right){ }^{14)}$ Oxygen-deficient strontium cobaltite $\left(\mathrm{SrCoO}_{2.5}\right)$ with Brownmillerite structure is known as insulating non-magnet, but it is changed into $\mathrm{SrCoO}_{3}$ with Perovskite structure, which is a ferromagnetic metal, by the oxidation. ${ }^{15)}$ Thus, the chemical modification of TMOs is the most reasonable way to demonstrate the functional switching devices, but the control of oxygen off-stoichiometry or protonation of TMOs is not typically utilized for solid-state devices because of imperative high-temperature heat treatment under an oxidative/reductive atmosphere. ${ }^{16)-20)}$ Meanwhile, the electrochemical redox reaction by the electrolysis in liquid electrolyte has been a facile method to achieve the chemical modification at room temperature (RT), ${ }^{21)}$ but such a device has not been realized because of the lack of a liquid-leakage-free electrolyte.

In 2010, H. Ohta et al. discovered a water-infiltrated nanoporous $12 \mathrm{CaO} \cdot 7 \mathrm{Al}_{2} \mathrm{O}_{3}$ glass (Calcium Aluminate with Nanopores, $\mathrm{CAN}$ ) as the liquid-leakage-free water. $^{22)}$ The $12 \mathrm{CaO}$ $7 \mathrm{Al}_{2} \mathrm{O}_{3}$ is a hygroscopic material and water vapor in air is automatically absorbed into the CAN film, cooperatively due to the capillary effect of the interconnected nanopores. "Liquid-leakagefree water" means the liquid water confined in the nano-space of the porous insulator film and no leakage of the liquid water occurs because of the large surface tension of the electrolyte and 
(a)

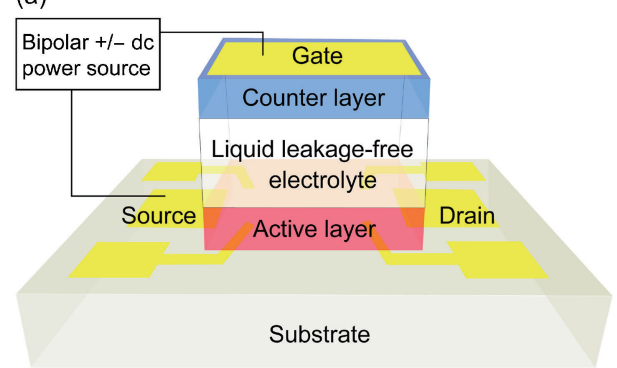

(b)

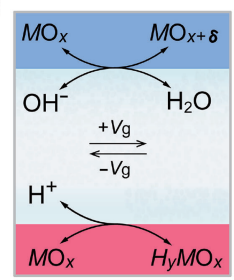

(c)

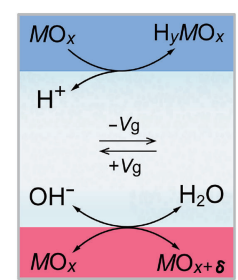

Fig. 1. Concept of an electrochemically switchable functional thin-film device. (a) Schematic of three terminal TFT structure composed of an active layer, a counter layer, a liquid-leakage-free electrolyte as a gate insulator, and source-drain-gate electrodes. Thin film of TMOs is used for the active layer. (b) Protonation/deprotonation. During positive gate voltage $\left(+V_{\mathrm{g}}\right)$ application, $\mathrm{H}^{+}$moves to the channel surface for the protonation of TMO layer. While, by applying $-V_{\mathrm{g}}$, the reverse reaction occurs for the deprotonation of TMO layer. (c) Oxidation/deoxidation. During $-V_{\mathrm{g}}$ application, $\mathrm{OH}^{-}$ion moves to the channel surface for the oxidation of TMO layer. While, by applying $+V_{\mathrm{g}}$, the reverse reaction occurs for the deoxidation of TMO layer.

nanopores. The CAN-gated TFT structure was fabricated on a $\mathrm{SrTiO}_{3}$ single crystal, where the gate voltage $\left(V_{\mathrm{g}}\right)$ application induces water electrolysis in the CAN gate insulator and the produced $\mathrm{H}^{+}\left(\mathrm{H}_{3} \mathrm{O}^{+}\right)$and hydroxyl $\left(\mathrm{OH}^{-}\right)$ion can be used to strongly accumulate and deplete electrons at the $\mathrm{SrTiO}_{3}$ channel surface. By applying the $V_{\mathrm{g}}$ at RT in air, the 2 dimensional electron gas layer with the sheet carrier electrons up to $\sim 10^{15} \mathrm{~cm}^{-2}$ can be formed at the surface of $\mathrm{SrTiO}_{3}$ single crystal and exhibits an unusually large thermopower, which is five times larger than that of the $\mathrm{SrTiO}_{3}$ bulk. ${ }^{23)}$ Thus, the CAN-gated TFT demonstrated a new approach to largely control the electronic states of TMOs by using the leakage-free water.

Meanwhile, the TFT structure with liquid-leakage-free electrolyte is considered as a kind of pseudo-solid-state electrochemical cell, where the ionized $\mathrm{H}^{+}$and $\mathrm{OH}^{-}$ion can be used to control the non-stoichiometry of the TMO channel. Figure 1 shows the schematic images of device structure and the possible operation mechanism for the electrochemically switchable device with three-terminal TFT structure using liquid-leakage-free electrolyte as the gate insulator. The device is composed of an active channel layer, a liquid-leakage-free electrolyte, a counter layer, and source-drain-gate electrodes [Fig. 1(a)]. The nano-gap parallel plate structure with an active layer and a counter layer should enable the reversible protonation/deprotonation or oxidation/ deoxidation of TMO layer in a high-electric field at RT. By applying positive $V_{\mathrm{g}}, \mathrm{H}^{+}$is attracted to channel surface, which can act to protonate the TMO layer [Fig. 1(b)]. While, the negative $V_{\mathrm{g}}$ application induces the reverse reaction for the deprotonation of TMO layer. On the other hand, by applying negative $V_{\mathrm{g}}, \mathrm{OH}^{-}$ion is attracted to channel surface, which can act to oxidize the TMO layer [Fig. 1(c)]. While, the positive $V_{\mathrm{g}}$ application induces the reverse reaction for the deoxidation of TMO layer. For both cases, the counter layer works as $\mathrm{H}^{+}$or $\mathrm{OH}^{-}$absorber, which maintains a better electrochemical balance and should improve the reversibility and reproducibility of device operation by suppressing the gas generation. Therefore, the optical, electronic, magnetic properties of TMO layer can be reversibly controlled by the alternative application of external $V_{\mathrm{g}}$.

In this paper, we review the electrochemically switchable functional thin-film devices based on TMOs of vanadium dioxide $\left(\mathrm{VO}_{2}\right), \mathrm{WO}_{3}$, and $\mathrm{SrCoO}_{x}$, realized by using the liquid-leakagefree electrolyte. (1) An infrared-transmittance tunable metalinsulator conversion device was demonstrated by using the leakage-free water for the on-demand protonation/deprotonation of $\mathrm{VO}_{2}$ film at $\mathrm{RT}^{24), 25)}$ (2) A transparent electrochromic metalinsulator switching device, that can simultaneously switch from colorless transparent/insulator state to a dark blue/metallic state by electrochemical protonation/deprotonation of a- $\mathrm{WO}_{3}$ film at RT, was demonstrated by using the leakage-free water. ${ }^{26)}$ (3) An electro-magnetic phase switching device, that can reversibly switch $\mathrm{SrCoO}_{x}$ from an insulator/non-magnet $\left(\mathrm{SrCoO}_{2.5}\right)$ to a metal/magnet $\left(\mathrm{SrCoO}_{3}\right)$, was demonstrated by using a newly developed 'leakage-free alkaline electrolyte', incorporated in an a- $\mathrm{NaTaO}_{3}$ nanopillar array film. ${ }^{27)}$

\section{Infrared-transmittance tunable metal-insulator conversion device ${ }^{24), 25)}$}

$\mathrm{VO}_{2}$ is a thermochromic material, which exhibits a metalinsulator (MI) transition at the temperature $\left(T_{\mathrm{MI}}\right)$ of $68^{\circ} \mathrm{C} .{ }^{28)}$ The MI transition accompanies the structural and electronic structure changes. The high-temperature phase $\left(T>T_{\mathrm{MI}}\right)$ has a tetragonal (rutile-type) structure, while the low-temperature phase $\left(T<T_{\mathrm{MI}}\right)$ has a monoclinic structure due to the formation of $\mathrm{V}$-ion dimer. ${ }^{29)}$ This structural transformation causes the electronic-structure reconstruction to open up a charge gap of $\sim 0.6 \mathrm{eV}$, where the electrical conductivity abruptly changes in the order of $10^{5}$ and optical transmittance dramatically changes only at infrared (IR) region while keeping the visible transparency. Thus, $\mathrm{VO}_{2}$ has a potential to demonstrate IR-transmittance tunable MI conversion device, which is expected as an advanced smart window: e.g. the device can selectively regulate thermal radiation from sunlight, and function as ON/OFF power switch to control the in-house temperature, which thus greatly reduces energy consumption including light expenses and cooling/heating loads.

Protonation of $\mathrm{VO}_{2}\left(\mathrm{H}_{x} \mathrm{VO}_{2}\right)$ is a powerful approach to control the electronic state from an insulator to a metal; the $\mathrm{H}^{+}$acts as a shallow donor and changes the valence state of $\mathrm{V}$ ion from $\mathrm{V}^{4+}$ to $\mathrm{V}^{3+}$, resulting in the reduction of $T_{\mathrm{MI}}$ below RT. ${ }^{30)}$ However, the protonation of $\mathrm{VO}_{2}$ required a high-temperature heating process, ${ }^{31-33)}$ which has restricted the device application. The liquid electrochemistry is the most appropriate route for the RT protonation, but the uptake amount of $\mathrm{H}^{+}$into $\mathrm{VO}_{2}$ was too small to modulate the MI transition (maximum $x$ in $\mathrm{H}_{x} \mathrm{VO}_{2}$ was 0.08 ) $^{31 \text { ) }}$ and the liquid electrolyte likely causes a leakage problem that limits the application in practical use. Thus, the RT-protonation driven on-demand $\mathrm{MI}$ conversion of $\mathrm{VO}_{2}$ by the solid-state device has been technologically important for a step toward practical application.

We first applied the TFT structure with liquid-leakage-free water to $\mathrm{VO}_{2}$ epitaxial film in order to investigate the RTprotonation-driven MI conversion. Figure 2(a) shows the schematic TFT structure, composed of an active $\mathrm{VO}_{2}$ layer, a CAN gate insulator, and source-drain-gate electrodes. The TFT structure (channel size: $800 \mu \mathrm{m}$ in length and $400 \mu \mathrm{m}$ in width) was fabricated on A-plane sapphire substrate by using stencil masks. A 20-nm-thick $\mathrm{VO}_{2}$ epitaxial film was deposited at $500^{\circ} \mathrm{C}$ under 
oxygen pressure $\left(P_{\mathrm{O} 2}\right)$ of $2.0 \mathrm{~Pa}$ by pulsed laser deposition (PLD). A 200-nm thick CAN film was deposited at RT under $P_{\mathrm{O} 2}$ of $5 \mathrm{~Pa}$ to realize a nanoporous structure. ${ }^{22}$ ) The relative density of the CAN film was $71 \%$ with respect to $2.92 \mathrm{~g} \mathrm{~cm}^{-3}$ of a dense a- $12 \mathrm{CaO} \cdot 7 \mathrm{Al}_{2} \mathrm{O}_{3}$ film. Metallic Ti films were used for sourcedrain-gate electrodes. Figure 2(b) shows a transmission electron microscopy (TEM) image for the cross-section of the device, which clearly shows the multi-layer structure of $\mathrm{Ti}(50 \mathrm{~nm}) / \mathrm{CAN}$ $(200 \mathrm{~nm}) / \mathrm{VO}_{2}(20 \mathrm{~nm})$ on sapphire substrate. Lighter spots with diameters of $10-20 \mathrm{~nm}$ indicates the high-density nanopores incorporated in the CAN film.

Figures 3(a) and 3(b) plot the $V_{\mathrm{g}}$ dependence of sheet resistance $\left(R_{\mathrm{S}}\right)$ and thermopower $(S)$ at RT for the $\mathrm{VO}_{2}$-TFT, respectively. The dependence was measured immediately after the bias application for $10 \mathrm{~min}$ at each $V_{\mathrm{g}}$ from +5 to $+35 \mathrm{~V}$. It should be noted that $S$-values can be used to easily evaluate the MI conversion because they basically reflect the energy differential of density of state (DOS) at Fermi energy and are sensitive to significant changes in the electronic structure of $\mathrm{VO}_{2}$ at $T_{\mathrm{MI}}{ }^{34), 35)}$ A double-digit decrease in $R_{\mathrm{s}}$ from $68 \mathrm{k} \Omega$ sq. ${ }^{-1}$ at the virgin state to $0.5 \mathrm{k} \Omega \mathrm{sq}^{-1}$ at $+35 \mathrm{~V}$ was observed and $|S|$-value drastically decreased form $200 \mu \mathrm{VK}^{-1}$ of insulating parent $\mathrm{VO}_{2}$ phase ${ }^{34)}$ to $43 \mu \mathrm{V} \mathrm{K}^{-1}$, which is close to that of metallic $\mathrm{H}_{x} \mathrm{VO}_{2}$ bulk $(\sim 35$

(a)

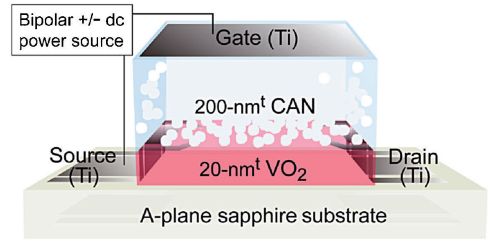

(b)

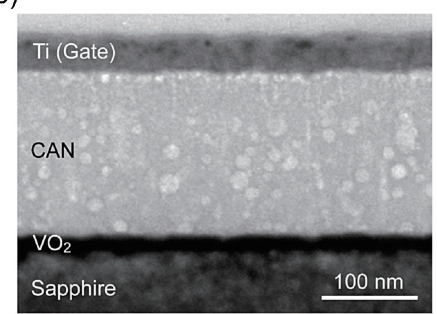

Fig. 2. (a) Schematic of the TFT structure composed of $\mathrm{VO}_{2}$ channel layer, water-infiltrated CAN gate insulator, and Ti source-drain-gate electrodes on A-plane sapphire substrate. (b) Cross-sectional TEM image of the $\mathrm{VO}_{2}$-TFT. Nanopores with $10-20 \mathrm{~nm}$ diameters are seen in the CAN film.
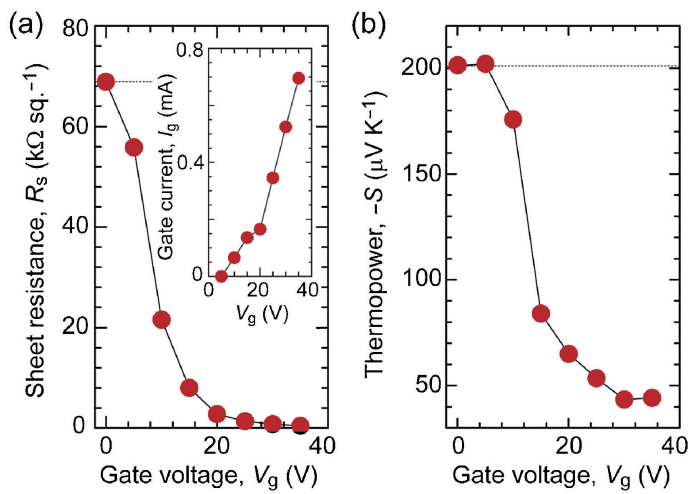

$\left.\mu \mathrm{V} \mathrm{K}^{-1}\right){ }^{33)}$ This result implies that the $\mathrm{H}^{+}$doping into insulating $\mathrm{VO}_{2}$ provides electrons to the conduction band, and the gradient of DOS becomes moderate, resulting in the consequent reduction of $|S|$-values, and the electronic structure finally transforms to that of metallic $\mathrm{H}_{x} \mathrm{VO}_{2}$. Therefore, the decrease in $R_{\mathrm{s}}$ and $|S|-$ values reflect a change in the electronic state from an insulator to a metal. The inset of Fig. 3(a) summarizes the $V_{\mathrm{g}}$ dependence of $I_{\mathrm{g}}$ during the bias application at each $V_{\mathrm{g}}$. The $I_{\mathrm{g}}$ increased with respect to $V_{\mathrm{g}}$ from +5 to $+35 \mathrm{~V}$. The similar correlation between $I_{\mathrm{g}}$ and $R_{\mathrm{s}}$ suggests that the $I_{\mathrm{g}}$ flowing in the device originates from the ion current of water-electrolysis in the CAN film and electrochemical protonation of $\mathrm{VO}_{2}$ layer. It should be noted that the metallic $\mathrm{H}_{x} \mathrm{VO}_{2}$ film is stable under ambient condition at RT, confirming the non-volatility due to the chemical reaction.

The MI transition of $\mathrm{VO}_{2}$ is strongly coupled with crystalstructure change from an insulating monoclinic phase to a metallic tetragonal phase, which is accompanied by the disappearance of V-ion dimer. ${ }^{29)}$ To validate the crystal structure change of metallic $\mathrm{H}_{x} \mathrm{VO}_{2}$ film, the structural analysis was performed by electron diffraction (ED) with TEM at RT. Figure 3(c) presents the nano-beam ED patterns observed at about $5 \mathrm{~nm}$ in depth from surface region of $\mathrm{VO}_{2}$ layer, before (upper panel) and after protonation (lower panel) with an incident electron beam along the [010] direction of $\mathrm{VO}_{2}$. The initial ED pattern is consistent with the monoclinic structure, where the diffraction spots of superstructures, indicated by arrows, originate from the formation of V-ion dimer. Following the protonation, the diffraction spots due to the superstructures disappear, demonstrating a transformation from the monoclinic to the tetragonal phase. This result clearly indicates that crystal structure was changed by RT protonation of $\mathrm{VO}_{2}$, which provides evidence of $\mathrm{MI}$ conversion.

The reversible protonation/deprotonation of $\mathrm{VO}_{2}$-TFT was examined by alternately applying positive and negative $V_{\mathrm{g}}$. Figure 3(d) presents the $R_{\mathrm{S}}$ at the OFF state versus number of gate pulses of +20 and $-20 \mathrm{~V}$ at RT, where each pulse duration was $1 \mathrm{~min}$. Upon the application of $V_{\mathrm{g}}$, reversible switching of $R_{\mathrm{S}}$ was realized. These results demonstrate the RT-protonationdriven on-demand $\mathrm{MI}$ conversion of $\mathrm{VO}_{2}$ film by using the leakage-free water.

We then demonstrate an IR-transmittance tunable MIconversion device by extending the TFT structure to a largearea $\mathrm{VO}_{2}$ film prepared on a glass substrate [Fig. 4(a)]. The device structure with the $2.0-\mathrm{mm}$-square $\mathrm{VO}_{2}$ channel was fabricated on an alkaline-free glass substrate (Corning ${ }^{\circledR}$ EAGLE $\left.\mathrm{XG}^{\circledR}\right)$. In order to fabricate the transparent device structure,
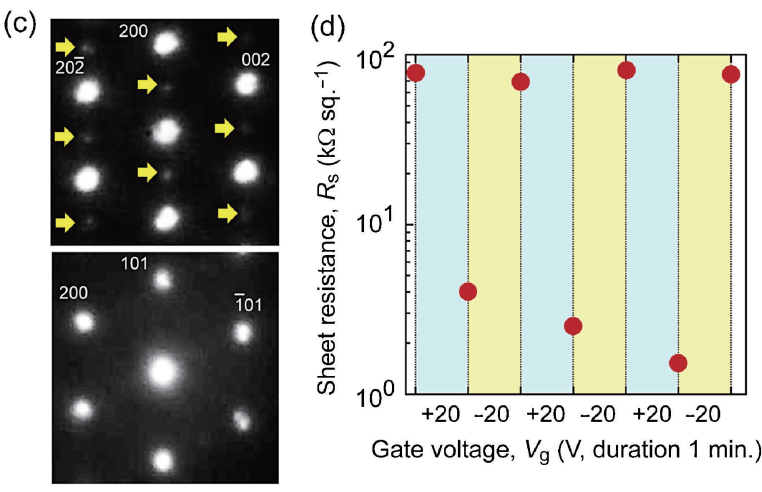

Fig. 3. (a) $R_{\mathrm{s}}$ as a function of $V_{\mathrm{g}}$ up to $+35 \mathrm{~V}$, where $R_{\mathrm{s}}$ was measured immediately after holding the $V_{\mathrm{g}}$ for 10 min. Inset shows $V_{\mathrm{g}}$ dependence of mean gate current $\left(I_{\mathrm{g}}\right)$ during the $V_{\mathrm{g}}$ application. (b) $S$ as a function of $V_{\mathrm{g}}$ up to $+35 \mathrm{~V}$. (c) Nano-beam ED patterns of $\mathrm{VO}_{2}$ film before (upper) and after protonation (lower). The main diffraction indices are noted above the corresponding diffraction spots. Arrows indicate the diffraction spots of superstructure due to $\mathrm{V}$-ion dimerization in monoclinic $\mathrm{VO}_{2}$. (d) Reversible MI conversion, where $\mathrm{VO}_{2}$ film was protonated at $V_{\mathrm{g}}=+20 \mathrm{~V}$, whereas deprotonation occurs at $V_{\mathrm{g}}=-20 \mathrm{~V}$. 
(a)

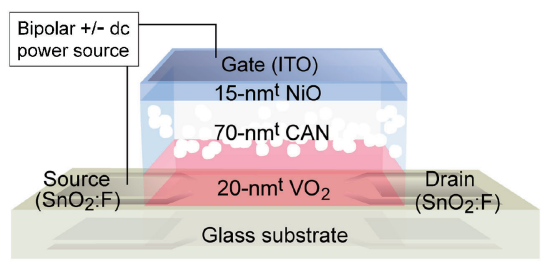

(b)

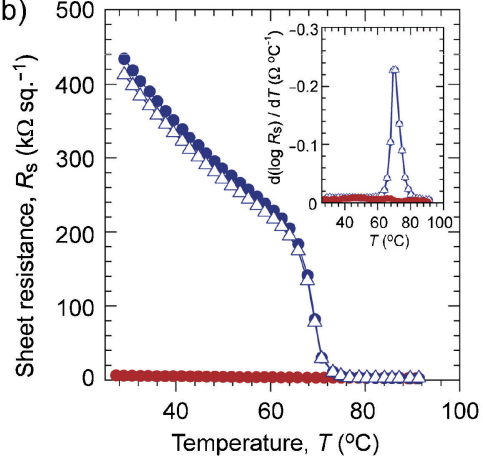

(c)

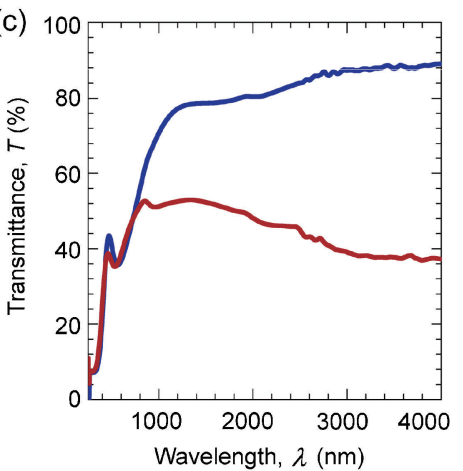

Fig. 4. (a) Schematic TFT structure consisting of transparent oxide thin films of $\mathrm{VO}_{2}$ channel layer, water-leakage-free CAN gate insulator, $\mathrm{NiO}$ counter layer/ITO gate electrode, and $\mathrm{SnO}_{2}: \mathrm{F}$ source-drain electrodes, on a glass substrate. (b) $R_{\mathrm{s}}-T$ curves measured before (blue closed circles) and after applying $V_{\mathrm{g}}$ alternately at $+12 \mathrm{~V}$ (protonation, red closed circles) and $-30 \mathrm{~V}$ (deprotonation, blue open triangles) at RT. Each curve was measured after switching the $V_{\mathrm{g}}$ off, where the $V_{\mathrm{g}}$ application time was $10 \mathrm{~s}$. Inset plots the temperature derivative curves of $\mathrm{d}\left(\log R_{\mathrm{s}}\right) / \mathrm{d} T$ in order to clearly visualize the MI transitions. The $R_{\mathrm{s}}$ modulation ratio $\left(R_{\mathrm{S}} /\right.$ initial $\left.R_{\mathrm{S}}\right)$ was 0.01 at $25^{\circ} \mathrm{C}$. (b) Optical transmittance spectra measured before (blue line) and after (red line) applying $V_{\mathrm{g}}=+12 \mathrm{~V}$.

which is essential to realize IR-transmittance modulation, all the films in the device were selected from the wide-gap oxide materials. A nickel oxide/indium tin oxide (NiO/ITO) bilayer film was used as the transparent counter/top gate electrode and F-doped $\mathrm{SnO}_{2}$ film $\left(\mathrm{SnO}_{2}: \mathrm{F}\right)$ was used as the source and drain electrodes. A positive $V_{\mathrm{g}}$ application between the gate and source electrodes induces electrochemical reactions such as protonation of the cathodic $\mathrm{VO}_{2}$ layer $\left(\mathrm{VO}_{2}+x \mathrm{H}^{+}+x \mathrm{e}^{-} \rightarrow \mathrm{H}_{x} \mathrm{VO}_{2}\right)$ and hydroxylation of the anodic $\mathrm{NiO}$ layer $\left(\mathrm{NiO}+\mathrm{OH}^{-} \rightarrow\right.$ $\left.\left.\mathrm{NiOOH}+\mathrm{e}^{-}\right) .{ }^{36}\right)$

The conversion of electrical conductivity and IR transmittance was investigated at RT in ambient atmosphere (relative humidity value was $\sim 30 \%$ at $25^{\circ} \mathrm{C}$ ). Figures 4 (b) and 4(c) summarize the opto-electronic properties of the $\mathrm{VO}_{2}$-TFT on a glass substrate. The temperature dependence of $R_{\mathrm{s}}$ [Fig. 4(b)] was measured before and after applying $V_{\mathrm{g}}$ of $+12 \mathrm{~V}$ (protonation) and $-30 \mathrm{~V}$ (deprotonation) for $10 \mathrm{~s}$ alternately at RT. The inset plots the temperature derivative curves of $\mathrm{d}\left(\log R_{\mathrm{S}}\right) / \mathrm{d} T$ to clearly visualize the $T_{\mathrm{MI}}$. At the initial state, the MI transition was observed at $T_{\mathrm{MI}}=70^{\circ} \mathrm{C}$, which is defined as the peak position in $\mathrm{d}\left(\log R_{\mathrm{S}}\right) /$ $\mathrm{d} T$ versus $T$, while it disappeared by applying $V_{\mathrm{g}}=+12 \mathrm{~V}$, indicating that the $\mathrm{VO}_{2}$ channel changes from an insulator to a metal at RT. The $R_{\mathrm{s}}-T$ curves were reversibly modulated and recovered to initial state by applying $V_{\mathrm{g}}=-30 \mathrm{~V}$, where the double-digit modulation of $R_{\mathrm{S}}$ was observed at RT. Then the optical transmission spectra [Fig. 4(c)] were measured. The initial device is transparent except for the weak absorption due to the transitions between the V $3 \mathrm{~d}$ bands with crystal-field splitting at the wavelength $(\lambda)>500 \mathrm{~nm}$ and also due to the thin-film interference. By applying $+12 \mathrm{~V}$, it shows an abrupt transmittance decrease in the IR region, where the transmittance modulation ratio $(\Delta T)$ at $\lambda=3000 \mathrm{~nm}$ was $49 \%$, while almost no change is seen in the VIS region. These results indicate that the modulation from IR transparent insulator to IR opaque metal was demonstrated by RT protonation/deprotonation of $\mathrm{VO}_{2}$ layer at $\mathrm{RT}$.

The present IR-transmittance tunable MI conversion device has several advantages. The device can be fabricated on a glass substrate, which is suitable for the application to glass window; the device fully transmits IR in the OFF state, whereas it does not transmit in the $\mathrm{ON}$ state. Meanwhile, the device can function as $\mathrm{ON} / \mathrm{OFF}$ power switch for electronic device to control the inhouse temperature. Moreover, the device can be operated by RTprotonation without sealing thanks to the leakage-free water; the all-solid-state structure can resolve the liquid-leakage problem, which is a beneficial point compared to the liquid-electrolyte gated devices. ${ }^{37)}$ The present result provides a potential gateway to the commercialization and ease of large-scale production; thus the present device will find the practical application for future energy saving technologies such as advanced smart windows.

\section{Electrochromic metal-insulator switching device ${ }^{26)}$}

$\mathrm{WO}_{3}$, a representative electrochromic (EC) material, is a widebandgap transparent insulator with $E_{\mathrm{g}}$ of $2.6-3.0 \mathrm{eV}{ }^{38)}$ Since the $\mathrm{WO}_{3}$ has the defective perovskite structure with space group $P 2_{1} / n$, in which A site in the $\mathrm{ABO}_{3}$ lattices is vacant, ${ }^{39)} \mathrm{H}^{+}$can be incorporated into the vacant site, i.e. the formation of tungsten bronze. In that case, it becomes an electrical conductor and opaque to visible light following the valence-state change of $\mathrm{W}$ ion from $\mathrm{W}^{6+}$ to $\mathrm{W}^{5+}$. 40) Thus, protonation/deprotonation of $\mathrm{WO}_{3}$ is a promising candidate for the realization of simultaneous switching between colorless/colored and insulating/conducting states in a non-volatile manner, which would be ideal for use in advanced display device: e.g. such EC-MI switching device can simultaneously switch the visible-color information and electrical information, which will lead to a novel information storage/ display device.

Until now, various types of $\mathrm{WO}_{3}$-based $\mathrm{EC}$ devices have been actively developed in the areas of energy-saving smart windows. ${ }^{41), 42)}$ However, simultaneous MI switching has not been utilized with EC switching because of their two-terminal, parallelplate electrode configuration. Although electrostatic charge modulation using three-terminal TFTs on $\mathrm{WO}_{3}$ films would be preferable for the simultaneous EC-MI switching, it is difficult to fully switch their coloring state due to the limited carrier-doping range and modulation thickness. There are some reports on $\mathrm{WO}_{3}$-TFTs using liquid electrolyte gating technique and the demonstration of EC-MI switching. ${ }^{43), 44)}$ However, since these TFTs require liquid electrolytes, they can not be applied for the practical applications without sealing.

We applyed the TFT-structure with leakage-free water as the gate insulator in order to demonstrate the EC-MI switching device with all solid-state structure. In addition, to show the potential for the practical use, the device was fabricated by RT process on a glass substrate (Corning ${ }^{\circledR}$ EAGLE $\mathrm{XG}^{\circledR}$ ). Figure 5(a) illustrates the TFT structure. All the films were prepared by PLD. The 
a- $\mathrm{WO}_{3}$ film was used as the active channel layer, because EC switching of a- $\mathrm{WO}_{3}$ film prepared on glass substrate at RT has been previously reported. ${ }^{45)}$ The gate insulator consisits of a CAN film as a leakage-free water. A NiO/ITO bilayer film was used as the gate transparent electrode, and ITO thin films were used as the transparent source and drain electrodes. Figures 5(b) and 5(c) show a bright-field scanning TEM (BF-STEM) image and energy dispersive spectroscopy (EDS) mapping of the cross-section of the device, which clearly visualizes the multi-layer structure composed of ITO $(20 \mathrm{~nm}) / \mathrm{NiO}(20 \mathrm{~nm}) / \mathrm{CAN}(300 \mathrm{~nm}) / \mathrm{a}-\mathrm{WO}_{3}$ $(80 \mathrm{~nm})$ on a glass substrate.

We fist evaluated the MI switching of the device by measuring $R_{\mathrm{S}}-T$ curves after applying and subsequently switching the $V_{\mathrm{g}}$ off (each $V_{\mathrm{g}}=+3,+5$, and $+10 \mathrm{~V}$ was applied for $20 \mathrm{~s}$ ). The relative humidity value, at which the device operation was tested, was $\sim 30 \%$ at $25^{\circ} \mathrm{C}$. All the $R_{\mathrm{s}}-T$ curves showed semiconducting behavior [Fig. 6(a)]; the exponential increase of $R_{\mathrm{S}}$ was observed with respect to temperature, which is consistent with the reports that electrical conductivity in a- $\mathrm{WO}_{3},{ }^{46)} \mathrm{a}-\mathrm{H}_{x} \mathrm{WO}_{3},{ }^{47)}$ and $\mathrm{a}-\mathrm{Na}_{x} \mathrm{WO}_{3}$ films, ${ }^{48)}$ follows the variable range hopping model between localized electronic states. The inset shows the activation energy $\left(E_{\mathrm{a}}\right)$, estimated from $\ln \left(1 / R_{\mathrm{s}}\right)$ vs. $1000 / T$ relation at $300-200 \mathrm{~K}$ range. The $E_{\mathrm{a}}$ largely decreased from $1.3 \times 10^{-1}$ to

(a)

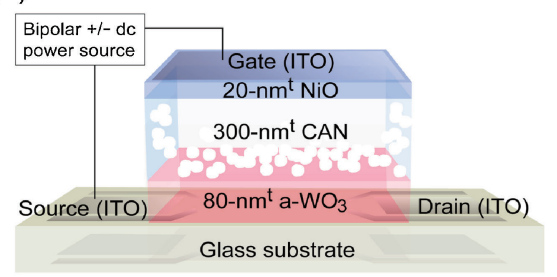

(b)

(c)
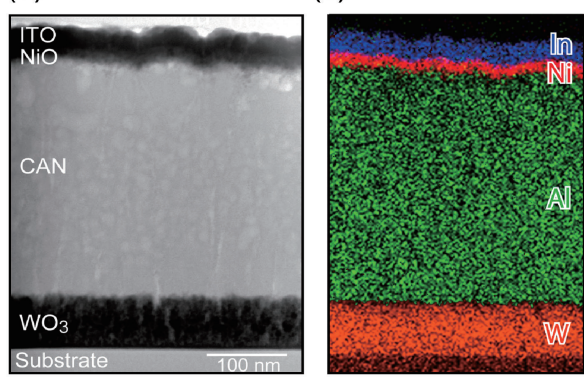

Fig. 5. (a) Schematic of TFT structure composed of a- $\mathrm{WO}_{3}(80 \mathrm{~nm})$, CAN $(300 \mathrm{~nm})$, and $\mathrm{NiO}(20 \mathrm{~nm})$ layers, on a glass substrate. Transparent ITO films are used for all the electrodes. $(\mathrm{b}, \mathrm{c})$ Cross-sectional BF-STEM image (b) and EDS mapping (c) of the device.
$4.3 \times 10^{-3} \mathrm{eV}$, and the $R_{\mathrm{s}}-T$ curve at $+10 \mathrm{~V}$ showed almost no temperature dependence. Considering that the minimum $E_{\mathrm{a}}$ of electrochemically prepared a- $\mathrm{H}_{x} \mathrm{WO}_{3}$ film $(x=0.32)$ was reported to be $5.0 \times 10^{-2} \mathrm{eV}^{47)}$ the doping concentration of $\mathrm{H}^{+}$is much higher in the present a- $\mathrm{H}_{x} \mathrm{WO}_{3}$ layer.

Figure 6(b) shows the repetitive $R_{\mathrm{s}}$ switching of a- $\mathrm{WO}_{3}$ layer at RT by applying $V_{\mathrm{g}}$ of $\pm 3, \pm 5$, and $\pm 10 \mathrm{~V}$, where the $V_{\mathrm{g}}$ application time was $20 \mathrm{~s}$ for protonation $\left(+V_{\mathrm{g}}\right)$ and $10 \mathrm{~s}$ for deprotonation $\left(-V_{\mathrm{g}}\right)$. The clear cyclability of $R_{\mathrm{s}}$ switching was observed for each $V_{\mathrm{g}}$, and the $R_{\mathrm{s}}$ modulation ratio largely depended on the applied $V_{\mathrm{g}}$; the ON/OFF ratio was $\sim 10^{3}$ for $\pm 3 \mathrm{~V}, \sim 10^{4}$ for $\pm 5 \mathrm{~V}$, and $\sim 10^{6}$ for $\pm 10 \mathrm{~V}$, respectively. The reversible and reproducible $R_{\mathrm{S}}$ switching with large $\mathrm{ON} / \mathrm{OFF}$ ratio was realized within a second time scale.

We then evaluated the EC switching of the device. Figure 6(c) shows the optical transmission spectra of the device in the initial state (gray line) and protonated states at $+3 \mathrm{~V}$ (blue line), $+5 \mathrm{~V}$ (green line), and $+10 \mathrm{~V}$ (red line). The transmission $(T)$ of the device was largely changed by the applied $+V_{\mathrm{g}}$. The initial device is mostly transparent in the visible light region. After the protonation, $T$ at $\lambda=700 \mathrm{~nm}$ was reduced to $24 \%$ of the initial state. Inset shows the device picture at the initial and protonated states $(+10 \mathrm{~V})$; the color clearly changes from colorlesstransparent to dark-blue. The EC-MI switching was simultaneously realized in the device.

The present EC-MI switching device can be reversibly switched from a colorless-transparent-insulator to a colored-metallicconductor within a short time $\sim 10 \mathrm{~s}$; the device has a potential to operate as a display device by changing visible color as well as an electronic memory device. In addition, the device can be prepared by RT process on a glass substrate, and the miniaturization of solid state TFT should be possible, leading to the highdensity memory device mounted on glass substrate. Thus the present device is expected to be a novel information display/ storage device on a glass window.

\section{Electro-magnetic phase switching device ${ }^{27)}$}

$\mathrm{SrCoO}_{x}\left(\mathrm{SCO}_{x}\right)$ is classically known to show different electromagnetic properties depending on the oxygen composition $(x)$, which can be varied in the range of 2.5-3.0, with keeping the crystallographic lattice structure. ${ }^{15), 49)}$ The most stable phase of $\mathrm{SCO}_{2.5}$ possesses an oxygen-vacancy ordered brownmillerite (BM-) structure. The fully-oxidized $\mathrm{SCO}_{3}$ phase, which can be synthesized under high oxygen-pressure and high temperature conditions, has simple perovskite $(\mathrm{P}-)$ structure. The parent $\mathrm{SCO}_{2.5}$ is an antiferromagnetic (AFM) insulator with trivalent $\mathrm{Co}^{3+}$, whereas the counterpart $\mathrm{SCO}_{3}$ is a ferromagnetic (FM)
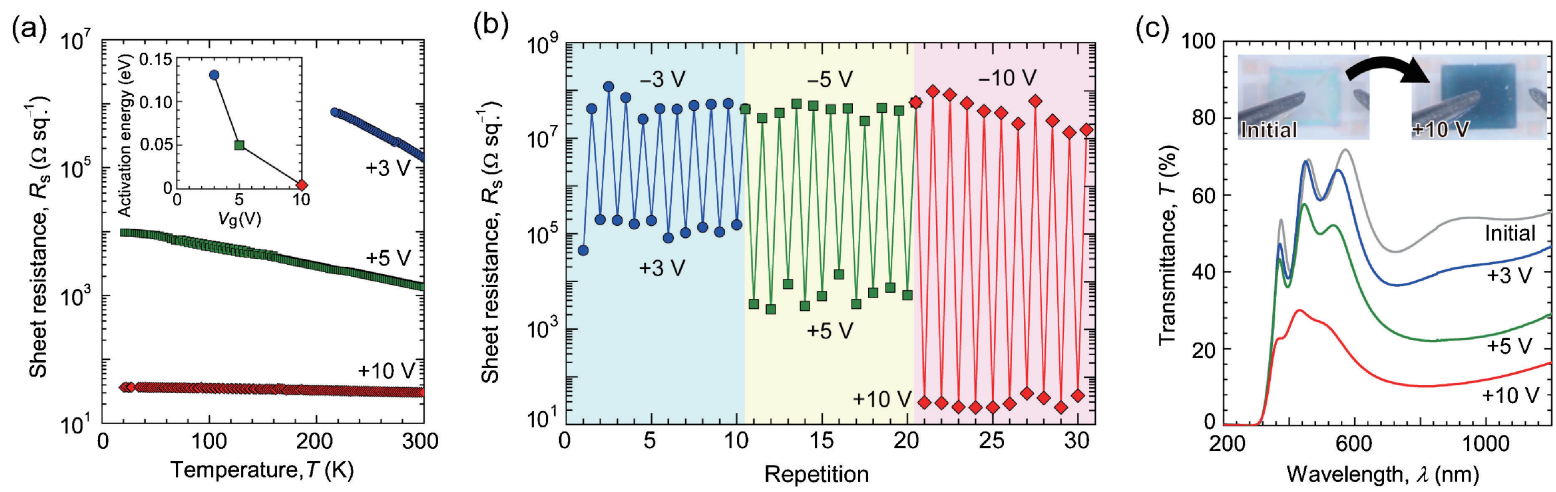

Fig. 6. EC-MI switching of a- $\mathrm{WO}_{3}$ TFT by applying various $V_{\mathrm{g}}= \pm 3, \pm 5, \pm 10 \mathrm{~V}$. (a) Temperature dependence of $R_{\mathrm{s}}$. (b) Reversible switching of $R_{\mathrm{S}}$ at RT. (c) Optical transmission spectra of the device. 
(a)
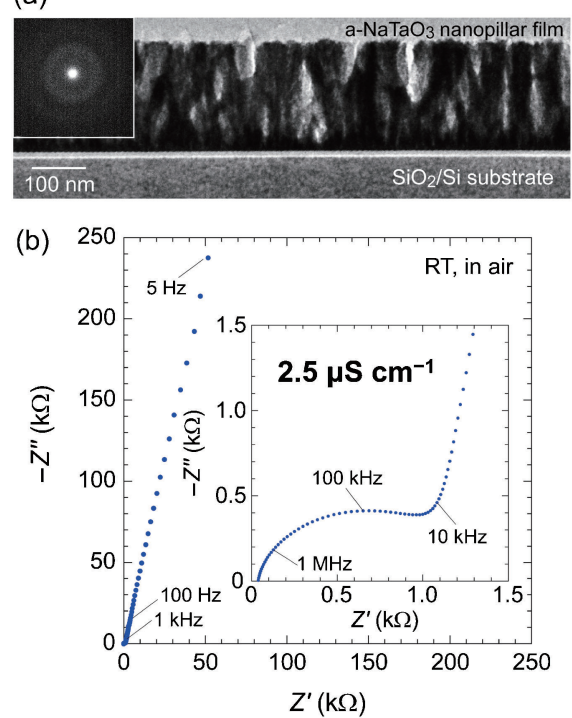

Fig. 7. (a) Cross-sectional TEM image of a 200 -nm-thick a- $\mathrm{NaTaO}_{3}$ film deposited on $\mathrm{SiO}_{2} / \mathrm{Si}$ substrate. A nanopillar array structure is seen. A broad halo is observed in the ED pattern. (b) Cole-Cole plot of the a- $\mathrm{NaTaO}_{3}$ nanopillar film $(950 \mu \mathrm{m} \times 800 \mu \mathrm{m})$ measured at $\mathrm{RT}$ in air.

metal with tetravalent $\mathrm{Co}^{4+}$. Thus, $\mathrm{SCO}_{x}$ has a potential to demonstrate an electro-magnetic phase switching device, whose electrical conductance (insulator/metal) and magnetism (nonmagnet/magnet) can be switched simultaneously. Such device is expected to be indispensable building blocks for future multiplex writing/reading of electric/magnetic signals.

However, the control of $x$ in $\mathrm{SCO}_{x}$ generally requires a heat treatment under oxidative/reductive atmosphere, ${ }^{50)}$ which is not preferable for devices operating at RT. The other is the use of an electrochemical redox reaction in a liquid electrolyte containing an alkali hydroxide as a salt at RT. ${ }^{51-54)}$ Although it would be reasonable for RT applications, the device cannot be used without sealing due to the leakage of the liquid alkaline electrolyte. Thus, a liquid-leakage-free alkaline electrolyte is essential to realize such electro-magnetic phase switching device.

We first developed a liquid-leakage-free alkaline electrolyte of a- $\mathrm{NaTaO}_{3}$ film with nano-sized pillar-array structure. We focused on $\mathrm{NaTaO}_{3}$, which is an electrical insulator with band gap of 4.0 $\mathrm{eV}$ and a promising photocatalyst for the $\mathrm{H}_{2}$ gas generation from water, ${ }^{55)}$ as the $\mathrm{Na}$ ion dissolved alkaline electrolyte. We finally found that the a-NaTaO 3 film, deposited by PLD under high $P_{\mathrm{O} 2}$ of $11 \mathrm{~Pa}$ at RT, works as an excellent liquid-leakage-free alkaline electrolyte. The a- $\mathrm{NaTaO}_{3}$ film is a nanoporous glassy oxide with a nanopillar (10-20 nm in diameter) array structure [Fig. 7(a)]. The film density is $3.3 \mathrm{~g} \mathrm{~cm}^{-3}$, which is $\sim 47 \%$ of fully dense a- $\mathrm{NaTaO}_{3}$ film $\left(7.0 \mathrm{~g} \mathrm{~cm}^{-3}\right)$ deposited under low $P_{\mathrm{O} 2}(<5 \mathrm{~Pa})$, indicating that the film has a large amount of space to absorb water vapor from the air. The Cole-Cole plot measured at RT in air (amplitude $= \pm 10 \mathrm{mV}$ ) of the a- $\mathrm{NaTaO}_{3}$ nanopillar array film is shown in Fig. 7(b). A semicircle is clearly seen in the highfrequency region due to mobile charge. The conductivity was calculated to be $2.5 \mu \mathrm{S} \mathrm{cm}^{-1}$, which is three orders of magnitude larger than $5 \mathrm{nS} \mathrm{cm}^{-1}$ of the dense a-NaTaO${ }_{3}$ film and two orders of magnitude larger than $55 \mathrm{nS} \mathrm{cm}^{-1}$ of ultra-pure water. ${ }^{56)}$ Water vapor in the air is incorporated into the $\mathrm{a}-\mathrm{NaTaO}_{3}$ nanopillar array film, and then, a small amount of $\mathrm{Na}^{+}$ions from the a- $\mathrm{NaTaO}_{3}$ nanopillars is dissolved into the adsorbed water; finally, the nanopores are filled with aqueous $\mathrm{NaOH}$ solution, which serves (a)
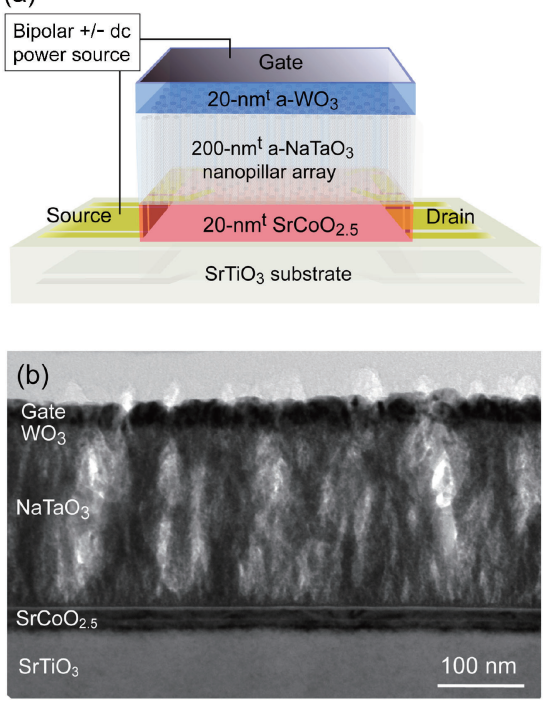

Fig. 8. (a) Schematic TFT structure composed of 20-nm-thick $\mathrm{SCO}_{2.5}$ active channel layer, leakage-free alkaline electrolyte (200-nm-thick a- $\mathrm{NaTaO}_{3}$ nanopillar array) as the gate insulator, 20-nm-thick a- $\mathrm{WO}_{3}$ / metal bilayer as the gate electrode. (c) Cross-sectional BF-STEM image of the device.

as a leakage-free alkaline electrolyte.

We then fabricated the TFT structure composed of a $20-\mathrm{nm}$ thick $\mathrm{SCO}_{2.5}$ epitaxial film as the active channel layer, a $200-\mathrm{nm}$ thick a-NaTaO${ }_{3}$ nanopillar array film as the gate insulator, and 20-nm-thick a- $\mathrm{WO}_{3}$ counter layer as the $\mathrm{H}^{+}$absorber/metal bilayer as the gate electrode [Fig. 8(a)]. This layer structure was confirmed by TEM image [Fig. 8(b)]. The channel length and channel width were 800 and $400 \mu \mathrm{m}$, respectively. The device switching was tested at RT in air without sealing.

Figures 9(a) and 9(b) summarize the electro-magnetic properties of $\mathrm{SCO}_{x}$-TFT. First, a negative $V_{\mathrm{g}}$ of $-3 \mathrm{~V}$ was applied for $3 \mathrm{~s}$. The $\mathrm{SCO}_{2.5}$ layer was brown in the virgin state but turned grey after the $V_{\mathrm{g}}$ application [Inset of Fig. 9(a)]. In the virgin state, the $\mathrm{SCO}_{2.5}$ layer showed insulating behaviour [Fig. 9(a), A]. In addition, the magnetic moment $(m)$ was negligibly small, with no magnetic transition in the measured temperature range [Fig. 9(b), A], indicating the AFM nature of $\mathrm{SCO}_{2.5}{ }^{15)}$ Upon applying a $V_{\mathrm{g}}$ of $-3 \mathrm{~V}, R_{\mathrm{s}}$ decreased by more than three orders of magnitude at RT, and the $\mathrm{SCO}_{x}$ layer showed metallic-temperature dependence [Fig. 9(a), B]. Furthermore, FM transition was observed at a Curie temperature of $275 \mathrm{~K}$ [Fig. 9(b), B], originating from the fully oxidized $\mathrm{SCO}_{3}$ phase. ${ }^{57)}$ The saturation magnetization at $10 \mathrm{~K}$ [inset of Fig. 9(b)] was $2.0 \mu_{\mathrm{B}} / \mathrm{Co}$, which is almost the same as that of bulk $\operatorname{SCO}_{3}\left(\sim 2.1 \mu_{\mathrm{B}} / \mathrm{Co}\right) .{ }^{51)}$ On the other hand, upon the application of $V_{\mathrm{g}}=+3 \mathrm{~V}$ for $3 \mathrm{~s}$, the $R_{\mathrm{s}}$ - and $m$-values recovered to the virgin state [Figs. 9(a) and 9(b), C]. We further tested the cyclability of the electro-magnetic phase switching [Fig. 9(c)]. Upon the application of $\pm 3 \mathrm{~V}$ for $2-3 \mathrm{~s}$ at RT in air, reversible switching of $R_{\mathrm{s}}\left(\mathrm{ON} / \mathrm{OFF}\right.$ ratio $\left.\sim 10^{3}\right)$ was realized.

Crystal structure evolution during the device operation can be seen in the X-ray reciprocal space mapping of $\mathrm{SCO}_{x}$ layer at the state of A and B [Fig. 9(d)]. By applying $V_{\mathrm{g}}=-3 \mathrm{~V}$, the diffraction spot of $\mathrm{BM}-\mathrm{SCO}_{2.5}$ changes to that of $\mathrm{P}-\mathrm{SCO}_{3}$, where the $c$-axis lattice constant of the perovskite unit cell steeply decreased from 0.393 to $0.379 \mathrm{~nm}$, maintaining the coherent epitaxial relationship with the $\mathrm{SrTiO}_{3}$ substrate. This result confirms that the entire region in the $\mathrm{SCO}_{x}$ layer was reversibly switched from AFM insulator $\left(\mathrm{BM}-\mathrm{SCO}_{2.5}\right)$ to $\mathrm{FM}$ metal $\left(\mathrm{P}-\mathrm{SCO}_{3}\right)$, with- 

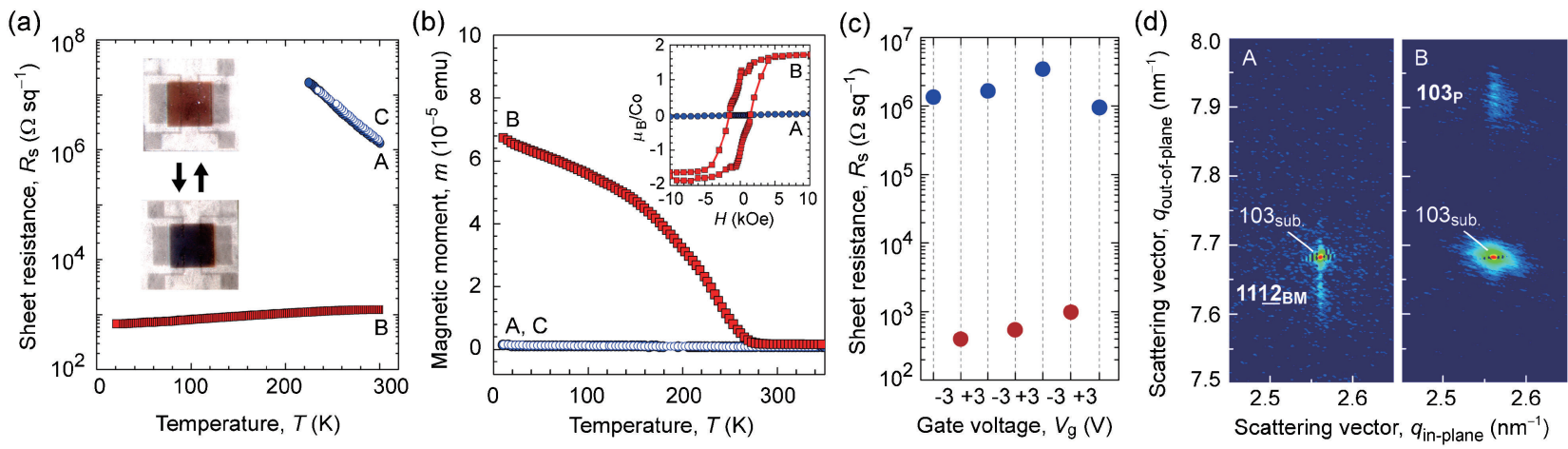

Fig. 9. (a) $R_{\mathrm{s}}-T$ curves of the $\mathrm{SCO}_{x}$ layer: (A) virgin state, (B) after applying a negative $V_{\mathrm{g}}=-3 \mathrm{~V}$, (C) subsequent $V_{\mathrm{g}}$ application of $+3 \mathrm{~V}$. The inset shows the picture of the device at the state of (A) and (B). (b) Temperature dependence of magnetic moment $(m)$ of the $\mathrm{SCO}_{x}$ layer at the states of A-C in (a), measured under $H=20$ Oe applied parallel to the in-plane direction. The inset shows a magnetic hysteresis loop at $10 \mathrm{~K}$ at states A and B. (c) Reversible switching of $R_{\mathrm{s}}$ under the application of $V_{\mathrm{g}}$ of $\pm 3 \mathrm{~V}$ with the duration time of 2-3 s. (d) X-ray reciprocal space mapping of $\mathrm{SCO}_{x}$ layer at the state of $\mathrm{A}$ and $\mathrm{B}$ in (a).

out destruction of the perovskite structure. ${ }^{50)}$

The resultant RT-operable electro-magnetic phase switching device showed excellent characteristics: reversible AFMinsulator/FM-metal switching of the $\mathrm{SCO}_{x}$ layer occurred electrically under a small DC voltage $( \pm 3 \mathrm{~V})$ within a very short time (2-3 s) at RT in air, i.e. the device is operable with two alkaline batteries $(3 \mathrm{~V})$ within the second time scale. The device can simultaneously switch electrical conductance and magnetism; such devices are expected to a new route for the high capacity information storage device, which memorizes both information 'A' (magnet) and 'B' (non-magnet) together with information ' 1 ' (conducting) and ' 0 ' (insulating).

\section{Summary}

We review the electrochemically switchable functional thinfilm devices based on TMOs of $\mathrm{VO}_{2}, \mathrm{WO}_{3}$, and $\mathrm{SCO}_{x}$, realized by using our liquid-leakage-free electrolytes. (1) An IRtransmittance tunable MI conversion device was demonstrated by using the leakage-free water for the on-demand protonation/ deprotonation of $\mathrm{VO}_{2}$ film at RT. ${ }^{24), 25)}$ (2) A transparent EC-MI switching device, that can simultaneously switch from colorless transparent/insulator state to a dark blue/metallic state by electrochemical protonation/deprotonation of a- $\mathrm{WO}_{3}$ film at $\mathrm{RT}$, was demonstrated by using the leakage-free water. ${ }^{26)}$ (3) An electromagnetic phase switching device, that can reversibly switch an insulator/non-magnet to a metal/magnet, was demonstrated by using a newly developed 'leakage-free alkaline electrolyte', incorporated in an a- $\mathrm{NaTaO}_{3}$ nanopillar array film. ${ }^{27)}$

The present device involving TMOs using liquid-leakage-free electrolytes has several merits as compared to classically known methods. Primarily, the device can be operated at RT, although high-temperature heating is required when utilizing simple $\mathrm{H}^{+}$ or $\mathrm{O}^{2-}$ diffusion in the TMOs under reductive/oxidative atmosphere. Further, the present device can be used for practical applications without sealing, as opposed to the known electrochemical devices such as alkaline batteries and electrolytic capacitors, which have the liquid leakage problem. This is an important advantage against recently developed EDLTs, which cannot be used without sealing as well. Furthermore, our liquidleakage-free electrolytes, such as CAN film and a- $\mathrm{NaTaO}_{3}$ film, are chemically stable rigid glassy solid, showing good reliability. These clearly indicate that the present approach utilizing the liquid-leakage-free electrolyte is epoch-making, providing a novel concept for the design of multifunctional switching devices. Since the present device electrochemically operates in a cycled process between a cathodic layer and an anodic layer, the water in the leakage-free electrolyte should not be lost during the device operation and the leakage-free electrolyte does not degrade after many cycles because of the no gas generation. However, it is considered that the humidity in air mainly affect the device operation. It is necessary to examine the device characteristics, cycled operations, and their stabilities for the devices kept under the controlled several conditions, which will be tested in the near future. In addition, the present devices simultaneously switch the two functions of optical, electronic, and magnetic properties. So, there is a limitation for their applications, as summarized in the manuscript. However, we consider that the present device has an ability of multilevel control of their properties by electrochemical modification of chemical composition, which will increase the information writing and reading approach and realize the high-capacity and multi-functional memory devices, compared to the simple semiconductor devices. If the materials, which have different threshold conditions for MI transition, chromic transition, and magnetic transition, are used for the channel layer, the further functionality is expected in the present type of device.

Acknowledgments The author gratefully thanks Mr. Kenji Endo, Mr. Yuki Suzuki, Mr. Takaki Onozato, Ms. Misako Hirono of Hokkaido University, and Mr. Taku Mizuno of Nagoya University for their great support on the device fabrication. Also, I would like to express my sincere thanks to Prof. Yuichi Ikuhara and Prof. Tetsuya Tohei for the TEM/STEM observation. This work was supported by Grant-in-Aid for Scientific Research A (25246023), Grant-in-Aid for Young Scientists A (15H05543), Grant-in-Aid for Challenging Exploratory Research (16K14377), and Grant-in-Aid for Scientific Research on Innovative Areas (25106007) from JSPS. TK was supported by PRESTO, JST.

\section{References}

1) J. Mannhart and D. G. Schlom, Nature, 430, 620-621 (2004).

2) E. Dagotto, Science, 309, 257-262 (2005).

3) H. Takagi and H. Y. Hwang, Science, 327, 1601-1602 (2010).

4) A. F. Wells, "Structural Inorganic Chemistry", 5th ed., Oxford University Press, Oxford (1984).

5) S. Lombardo, J. H. Stathis, B. P. Linder, K. L. Pey, F. Palumbo and C. H. Tung, J. Appl. Phys., 98, 121301 (2005).

6) J. Robertson, Eur. Phys. J. Appl. Phys., 28, 265-291 (2004).

7) C. H. Ahn, A. Bhattacharya, M. Di Ventra, J. N. Eckstein, C. Daniel Frisbie, M. E. Gershenson, A. M. Goldman, I. H. Inoue, J. Mannhart, A. J. Millis, A. F. Morpurgo, D. Natelson and J.-M. Triscone, Rev. Mod. Phys., 78, 1185-1212 (2006). 
8) K. Ueno, S. Nakamura, H. Shimotani, A. Ohtomo, N. Kimura, T. Nojima, H. Aoki, Y. Iwasa and M. Kawasaki, Nat. Mater., 7, 855-858 (2008).

9) Y. Yamada, K. Ueno, T. Fukumura, H. T. Yuan, H. Shimotani, Y. Iwasa, L. Gu, S. Tsukimoto, Y. Ikuhara and M. Kawasaki, Science, 332, 1065-1067 (2011)

10) M. Nakano, K. Shibuya, D. Okuyama, T. Hatano, S. Ono, M. Kawasaki, Y. Iwasa and Y. Tokura, Nature, 487, 459-462 (2012).

11) T. Katase, H. Hiramatsu, T. Kamiya and H. Hosono, Proc Natl. Acad. Sci. USA, 111, 3979-3983 (2014).

12) M. A. Hayward and M. J. Rosseinsky, Chem. Mater., 12, 2182-2195 (2000)

13) J. B. Goodenough, Rep. Prog. Phys., 67, 1915-1993 (2004).

14) C. G. Granquist, Sol. Energy Mater. Sol. Cells, 60, 201-262 (2000).

15) T. Takeda, Y. Yamaguchi and H. Watanabe, J. Phys. Soc. Jpn., 33, 970-972 (1972).

16) J. Mizusaki, S. Yamaguchi, K. Fueki and A. Ishikawa, Solid State Ionics, 12, 119-124 (1984).

17) Z. Shao and S. M. Haile, Nature, 431, 170-173 (2004).

18) R. Merkle and J. Maier, Angew. Chem. Int. Ed., 47, 3874-3894 (2008).

19) A. M. Chippindale, P. G. Dickens and A. V. Powell, J. Solid State Chem., 93, 526-533 (1991).

20) V. N. Andreev, V. M. Kapralova and V. A. Klimov, Phys. Solid State, 49, 2318-2322 (2007).

21) J-C. Grenier, A. Wattiaux, J-P. Doumerc, P. Dordor, L. Fournes, J-P. Chaminade and M. Pouchard, J. Solid State Chem., 96, 20-30 (1992).

22) H. Ohta, Y. Sato, T. Kato, S. W. Kim, K. Nomura, Y. Ikuhara and H. Hosono, Nat. Commun., 1, 118 (2010).

23) H. Ohta, T. Mizuno, S. Zheng, T. Kato, Y. Ikuhara, K. Abe, H. Kumomi, K. Nomura and H. Hosono, Adv. Mater, 24, 740 (2012).

24) T. Katase, K. Endo, T. Tohei, Y. Ikuhara and H. Ohta, $A d v$. Electron. Mater., 1, 1500063 (2015).

25) T. Katase, K. Endo and H. Ohta, APL Mater., 5, 056105 (2017).

26) T. Katase, T. Onozato, M. Hirono, T. Mizuno and H. Ohta, Sci. Rep., 6, 25819 (2016).

27) T. Katase, Y. Suzuki and H. Ohta, Adv. Electron. Mater., 2, 1600044 (2016).

28) F. J. Morin, Phys. Rev. Lett., 3, 34-36 (1959).

29) J. B. Goodenough, J. Solid State Chem., 3, 490-500 (1971).

30) J. Wei, H. Ji, W. Guo, A. H. Nevidomskyy and D. Natelson, Nat. Nanotechnol., 7, 357-362 (2012).

31) A. M. Chippindale, P. G. Dichens and A. V. Powell, J. Solid State Chem., 93, 526-533 (1991).

32) V. Andreev, V. Kapralova and V. Klimov, Phys. Solid State, 49, 2318-2322 (2007).

33) C. Wu, F. Feng, J. Feng, J. Dai, L. Peng, J. Zhao, J. Yang, C. Si, Z. Wu and Y. Xie, J. Am. Chem. Soc., 133, 13798-13801
(2011).

34) T. Katase, K. Endo and H. Ohta, Phys. Rev. B, 90, 161105 (2014).

35) T. Katase, K. Endo and H. Ohta, Phys. Rev. B, 92, 035302 (2015).

36) X. H. Xia, J. P. Tu, J. Zhang, X. L. Wang, W. K. Zhang and H. Huang, Sol. Energy Mater. Sol. Cells, 92, 628-633 (2008).

37) M. Nakano, K. Shibuya, N. Ogawa, T. Hatano, M. Kawasaki, Y. Iwasa and Y. Tokura, Appl. Phys. Lett., 103, 153503 (2013).

38) P. P. González-Borrero, F. Sato, A. N. Medina, M. L. Baesso, A. C. Bento, G. Baldissera, C. Persson, G. A. Niklasson, C. G. Granqvist and A. Ferreira da Silva, Appl. Phys. Lett., 96, 061909 (2010).

39) H. Zheng, J. Z. Ou, M. S. Strano, R. B. Kaner, A. Mitchell and K. Kalantar-zadeh, Adv. Funct. Mater., 21, 2175-2196 (2011).

40) P. G. Dickens and M. S. Whittingham, Q. Rev. Chem. Soc., 22, 30-44 (1968).

41) C. G. Granqvist, Sol. Energy Mater. Sol. Cells, 92, 203-208 (2008).

42) S. K. Deb, Sol. Energy Mater. Sol. Cells, 92, 245-258 (2008)

43) M. J. Natan, T. E. Mallouk and M. S. Wrighton, J. Phys. Chem., 91, 648-654 (1986).

44) P. Barquinha, S. Pereira, L. Pereira, P. Wojcik, P. Grey, R. Martins and E. Mortunato, Adv. Electron. Mater., 1, 1500030 (2015).

45) S. K. Deb, Philos. Mag., 27, 801-822 (1973).

46) C. Bechinger, S. Herminghaus and P. Leiderer, Thin Solid Films, 239, 156-160 (1994).

47) R. S. Crandall and B. W. Faughnan, Phys. Rev. Lett., 39, 232235 (1977).

48) I. C. Lekshmi, A. Gayen, V. Prasad, S. V. Subramanyam and M. S. Hegde, Mater. Res. Bull., 37, 1815-1823 (2002).

49) T. Takeda and H. Watanabe, J. Phys. Soc. Jpn., 33, 973-978 (1972).

50) H. Jeen, W. S. Choi, M. D. Biegalski, C. M. Folkman, I-C Tung, D. D. Fong, J. W. Freeland, D. Shin, H. Ohta, M. F. Chisholm and H. N. Lee, Nat. Mater, 12, 1057-1063 (2013).

51) P. Bezdicka, A. Wattiaux, J. C. Grenier, M. Pouchard and P. Hagenmuller, Z. Anorg. Allg. Chem., 619, 7-12 (1993).

52) A. Nemudry, P. Rudolf and R. Schöllhorn, Chem. Mater, 8 , 2232-2238 (1996)

53) N. Ichikawa, M. Iwanowska, M. Kawai, C. Calers, W. Paulusbd and Y. Shimakawa, Dalton Trans., 41, 10507-10510 (2012).

54) O. T. Tambunan, M. Y. Lee, D. H. Kim, K. J. Parwanta and C. U. Jung, J. Korean Phys. Soc., 64, 1845-1848 (2014).

55) H. Kato and A. Kudo, J. Phys. Chem. B, 105, 4285-4292 (2001).

56) T. S. Light, S. Licht, A. C. Bevilacqua and K. R. Morash, Electrochem Solid St., 8, E16-E19 (2005).

57) S. Balamurugan, K. Yamaura, A. B. Karki, D. P. Young, M. Arai and E. Takayama-Muromachi, Phys. Rev. B, 74, 172406 (2006).

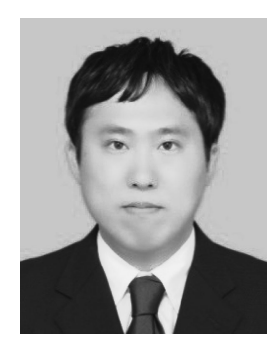

Takayoshi Katase is currently Associate Professor of Laboratory for Materials and Structures, Institute of Innovative Research, Tokyo Institute of Technology, and also PRESTO researcher, Japan Science and Technology Agency ("Scientific Innovation for Energy Harvesting Technology" area). He received his B.E. from Department of Inorganic Materials, School of Engineering, Tokyo Institute of Technology in 2007 and received his M.E. from Department of Materials Science and Engineering, Interdisciplinary Graduate School of Science and Engineering, Tokyo Institute of Technology in 2009, and his Ph.D. from Department of Materials Science and Engineering, Interdisciplinary Graduate School of Science and Engineering, Tokyo Institute of Technology in 2012 (Dissertation title: Heteroepitaxial Growth and Superconducting Properties of Iron Pnictide Thin Films, Supervisor: Prof. Hideo Hosono). He worked as a Postdoctoral researcher at Frontier Research Center, Tokyo Institute of Technology in 2012-2013. He was an Assistant Professor of Research Institute for Electronic Science, Hokkaido University in 2013-2017. 